\title{
Gaia CCDs: charge transfer inefficiency measurements between five years of flight
}

Ahmed, Saad, Hall, David, Crowley, Cian, Skottfelt, Jesper, Dryer, Ben, et al.

Saad Ahmed, David Hall, Cian Crowley, Jesper Skottfelt, Ben Dryer, George Seabroke, Jose Hernandez, Andrew Holland, "Gaia CCDs: charge transfer inefficiency measurements between five years of flight," Proc. SPIE 11454, XRay, Optical, and Infrared Detectors for Astronomy IX, 114540S (13 December 2020); doi: 10.1117/12.2562162 


\title{
Gaia CCDs: Charge transfer inefficiency measurements between 5 years of flight
}

\author{
Saad Ahmed ${ }^{\mathrm{a}}$, David Hall ${ }^{\mathrm{a}}$, Cian Crowley ${ }^{\mathrm{b}}$, Jesper Skottfelt ${ }^{\mathrm{a}}$, Ben Dryer ${ }^{\mathrm{a}}$, George Seabroke , $^{\mathrm{c}}$ \\ Jose Hernandez ${ }^{\mathrm{d}}$, and Andrew Holland ${ }^{\mathrm{a}}$ \\ ${ }^{a}$ Centre for Electronic Imaging, SPS, The Open University, Milton Keynes, MK7 6AA, UK \\ ${ }^{b} \mathrm{HE}$ Space Operations BV for ESA/ESAC, Camino Bajo del Castillo s/n, 28691 Villanueva de \\ la Cañada, Spain \\ ${ }^{c}$ Mullard Space Science Laboratory, University College London, Surrey, RH5 6NT, UK \\ ${ }^{d}$ ESA, European Space Astronomy Centre, Camino Bajo del Castillo s/n, 28691 Villanueva de \\ la Cañada, Spain
}

\begin{abstract}
The European Space Agency's Gaia spacecraft was launched in December 2013 and has been in orbit at the Earth-Sun Lagrange point 2 (L2) for over 6 years. The spacecraft measures the positions, distances, space motions and many other physical characteristics of around one billion stars in the Milky Way and beyond. It has a focal plane of 106 Charge-Coupled Devices (CCDs) which have all been performing well but have been measuring a small but quantifiable degradation in performance in time due to Non-Ionizing Energy Loss (NIEL) damage from interstellar radiation. This NIEL damage produces trap defects which can capture charge from signals and reduces the quality of the data. Gaia's original mission lifetime was planned to be around 5 years and the pre-flight testing and radiation damage analysis was tailored around those timescales as well as with the projected solar activity before launch. Closer to the time of launch and during Gaia's years of orbit, it has been noted that the solar activity was lower than what was initially predicted. From the previous analysis of in-flight data in 2016, it was calculated that Gaia was experiencing an order of magnitude less radiation damage than was predicted. This paper describes the analysis of charge calibration data and corresponding Charge Transfer Inefficiency (CTI) measurements from the in-flight CCDs, both near the beginning of the mission and after more than 5 years in orbit to quantify the radiation damage impact. These sets of results can be compared with those from the pre-flight tests which can be used to evaluate and understand the differences between the on-ground and in-flight results.
\end{abstract}

Keywords: CCD, Gaia, Radiation Damage, Silicon, Protons, Shielding, NIEL

\section{INTRODUCTION}

The Gaia spacecraft is one of the European Space Agency's missions from the Horizon 2000 Plus programme, successfully launched in 2013. Its goal is to make the largest astrometric and stereoscopic catalogue of astronomical objects in the galaxy, mapping out around $1 \%$ of the entire Milky Way and producing a database that comprises of more than one billion stars. Rotating with a period of approximately six hours, Gaia scans the sky with its two optical telescopes making repeated observations of stars, galaxies, quasars and other objects. Gaia is designed in such a way so as to measure numerous properties for every observation such as photometry, and medium and low-resolution spectra, in addition to position and parallax. ${ }^{1,2}$ To date, two Data Releases have already been released from the large quantities of data collected by the spacecraft with a third Data Release* currently being prepared. $^{2,3}$ To fulfil its objectives, Gaia has a focal plane of 106 Charge-Coupled Devices (CCDs). Figure 1 illustrates a schematic of the focal plane with the devices colour-coded by instruments.

Further author information: (Send correspondence to Saad Ahmed)

Saad Ahmed. E-mail: saad.ahmed@open.ac.uk,www.open.ac.uk/science/research/cei

${ }^{*}$ For the latest updates on the data releases and the mission in general see, https://www.cosmos.esa.int/web/gaia/data

X-Ray, Optical, and Infrared Detectors for Astronomy IX, edited by Andrew D. Holland,

James Beletic, Proc. of SPIE Vol. 11454, 114540S · (c) 2020 SPIE

CCC code: $0277-786 \mathrm{X} / 20 / \$ 21 \cdot$ doi: $10.1117 / 12.2562162$

Proc. of SPIE Vol. 11454 114540S-1 


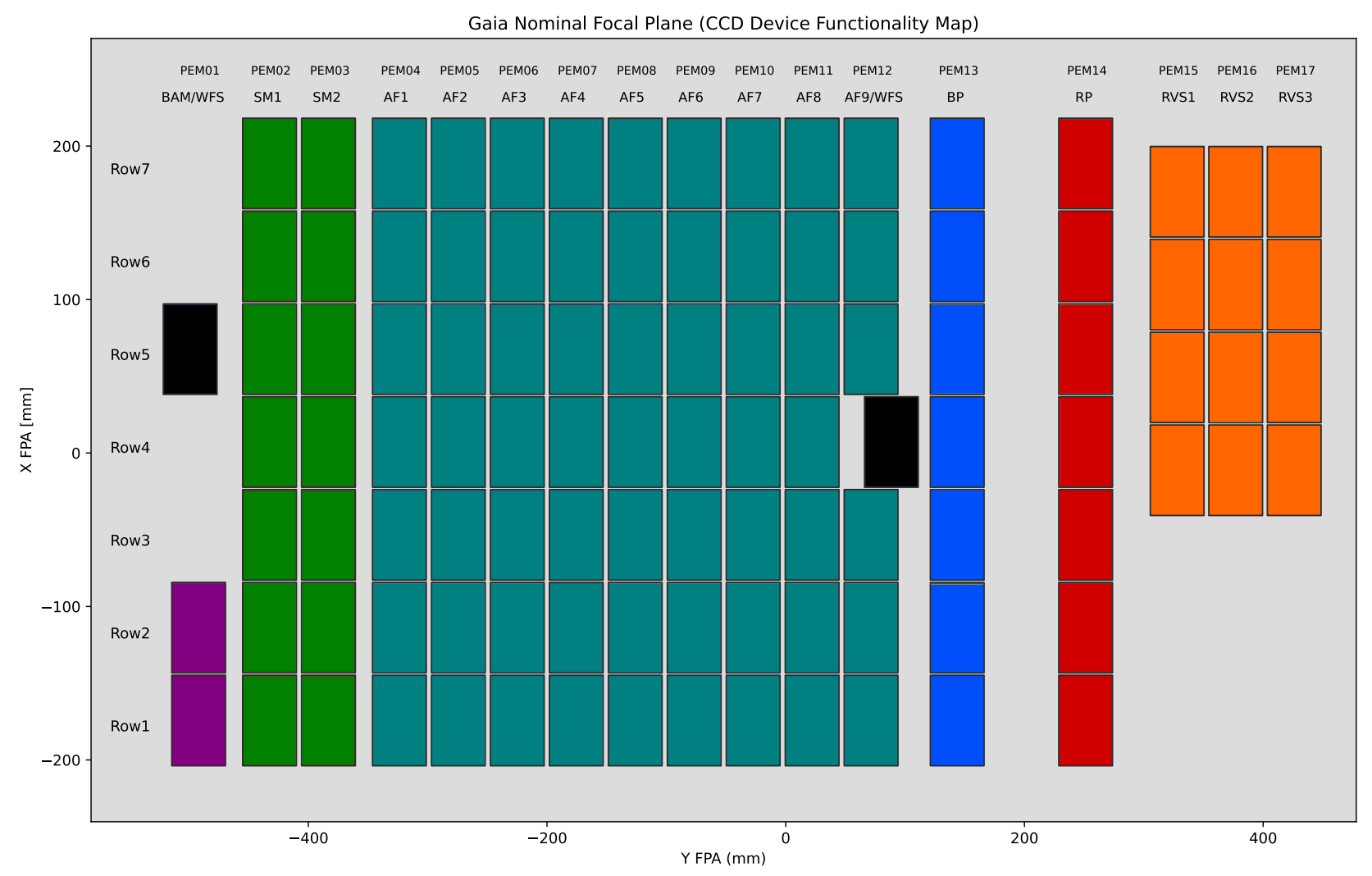

Figure 1. The Gaia focal plane with 106 large-format CCDs, colour-coded by instruments. The colour designations are as follows: black for the Wavefront Sensors (WFS), purple for the Basic Angle Monitors (BAM), green for the Sky Mappers $(\mathrm{SM})$, turquoise for the Astrometric Field (AF), blue for the Blue Photometer (BP), red for the Red Photometer (RP) and orange for the Radial Velocity Spectrometer (RVS).

\subsection{CCDs and the focal plane}

Gaia's CCDs were custom-designed and manufactured by Teledyne e2v and were derived from the CCD91-72 model. They are back-illuminated, full-frame n-channel devices with an image area of $4500 \times 1966$ pixels, with each pixel having a size of $10 \mu \mathrm{m} \times 30 \mu \mathrm{m}$. Gaia's CCDs operate in a unique Time-Delayed Integration (TDI) mode where the CCDs collect and transfer charge as the object images passes over them, synchronous with the spacecraft spin rate. The parallel or ALong-scan (AL) transfer rate corresponds to a period of $982.8 \mu$ s. The integration time per CCD is 4.42 seconds, corresponding to 4500 TDI AL lines. Due to the TDI operation, signals need to be transferred quickly through the serial register, in the ACross-Scan (AC) direction, which has a flush frequency of $10 \mathrm{MHz}$ to transfer signals to the readout node. The signals are then read out at different readout frequencies, unique to each different instrument. ${ }^{2}$

The Gaia CCDs also contain two unique design features to reduce the impact of radiation damage and charge trapping, a Charge Injection (CI) structure and a Supplementary Buried Channel (SBC). The CI structure periodically injects charge at regular intervals in most of the CCDs; this fills the traps to reduce the charge loss from signals. The Supplementary Buried Channel (SBC) is a notch in the buried channel region that confines small charge packets to a smaller volume so that they encounter fewer traps. While all of Gaia's CCDs were manufactured with these features and with identical chip architectures, they were also manufactured in three different variants, each optimised to improve their quantum efficiency in different wavelength regimes to fulfill alternate objectives.

The three CCD variants that were manufactured with the Gaia CCD design were the Astrometric Field (AF), 
Blue Photometer (BP) and Red Photometer (RP) variant CCDs. The AF CCDs were built on $25 \mu \mathrm{m}$ standard silicon thinned to $16 \mu \mathrm{m}$ with a resistivity of $100 \Omega \mathrm{cm}$, and have an Anti-Reflection (AR) coating centred at $650 \mathrm{~nm}$. These variants were used on the all the devices of the Astrometric Field as well as the Sky Mapper (SM) CCDs and the Wave-Front Sensors (WFS). The BP CCDs were also made with $16 \mu \mathrm{m}, 100 \Omega \mathrm{cm}$ resistivity standard silicon but have an AR-coating that was centred on 360nm, towards the bluer wavelengths. Only the seven CCDs on the Blue Photometer instrument used this device variant. The RP CCDs were made for the instruments that needed to be more sensitive to redder wavelengths. Due to this, they were manufactured with a thickness of $40 \mu \mathrm{m}$, using deep-depleted silicon that had a resistivity of $1500 \Omega \mathrm{cm}$ and had an AR-coating centred at $750 \mathrm{~nm}$. These variants were used in the seven CCDs of the Red Photometer Instrument, the 12 CCDs of the Radial Velocity Spectrometer (RVS), as well as the Basic-Angle Monitor (BAM) CCDs. ${ }^{4,5}$

The pre-flight testing and the manufacturing of Gaia's devices took place over several years. As a result, devices manufactured at different dates were used, both for performing radiation testing and sector analysis, as well as for the in-flight devices. In addition to this, because of the comparatively larger number of CCDs present on the Gaia focal plane as compared to any other space mission, all the in-flight devices were manufactured at different times and came from different batches of silicon. In a previous study, it was found that the CCDs that were manufactured after 2004 may contain a reduced SBC Full Well Capacity as compared to earlier devices. ${ }^{6}$ In order to check for any correlations between device performance in the results of this paper and the time of production, Figure 2 was produced which illustrates the corresponding year of manufacture of the in-flight devices.

\section{DISPLACEMENT DAMAGE IN A RADIATION ENVIRONMENT}

Gaia orbits Lagrange Point 2 (L2), the same destination of several other future space missions such as Euclid and the James Webb Space Telescope (JWST). Due to this, understanding the performance of Gaia at this location can provide key insights into the expected behaviour and performances of other spacecraft at this location. The



Figure 2. The year of manufacture of all the in-flight devices on the Gaia focal plane. 
L2 saddle point is a stable gravitational point that is at a location that is $\sim 1.5$ million $\mathrm{km}$ from the Earth, and co-rotates with the Earth when it orbits around the Sun. Gaia moves around L2 in a Lissajous-type orbit which offers a stable thermal environment and a relatively calmer radiation environment as compared to an Earth-bound orbit. In the early two years of the mission, it was noted that the L2 environment had not yielded any unexpected results. ${ }^{2}$

Due to the predicted radiation environment in this region, as well as the high-precision measurement requirements of the mission, the increase of Charge Transfer Inefficiency (CTI) in the CCDs from non-ionizing radiation damage was identified in the pre-launch studies as a major factor that would impact the useful scientific output of the mission. The main sources of radiation at L2 were identified as particles from solar events and Galactic Cosmic Rays (GCRs). When high energy particles hit the detector, they can knock atoms out of the silicon lattice which forms vacancies or absences of silicon atoms. These vacancies diffuse through the lattice and can form stable defects with either other vacancies or doping or impurity atoms. These defects form new energy levels between the valence and conduction bands and form an electron 'trap' in the silicon. Traps can capture electrons from passing charge packets and release them on probabilistic timescales, as governed by the Shockley-Read-Hall equations. Different trap defects emit at different times which can be altered by the operating temperature of the device. ${ }^{7,8}$ Gaia's operating temperature is $163.15 \mathrm{~K}$; chosen as a balance between the CTI contributions from the $\mathrm{AL}$ and $\mathrm{AC}$ transfer speeds. ${ }^{9}$

\section{PREVIOUS STUDIES}

Before launch, numerous studies were conducted to quantify and determine all the factors that would affect the CCD performance. As a precursor to the radiation campaigns that were performed to investigate the impact of radiation damage, Airbus Defence and Space (formerly EADS Astrium) simulated a prediction for the $10 \mathrm{MeV}$ equivalent NIEL dose across the focal plane in 2006. Using the predicted level of solar activity at the time, it was calculated that most of the AF CCDs (the ones most exposed to the radiation) would receive a NIEL dose of $3.1 \times 10^{9} \mathrm{p}^{+} / \mathrm{cm}^{2} 10 \mathrm{MeV}$ equivalent protons. A $90 \%$ confidence interval was implemented when making this prediction. ${ }^{10}$ From these results, the on-ground radiation campaigns performed CCD irradiations at slightly higher levels to overestimate the amount of radiation damage. Many useful insights and CTI mitigation strategies were derived from the following experiments and measurements which were implemented for the mission. These include the setting of the $163.15 \mathrm{~K}$ operating temperature to optimise CTI between parallel and serial transfer, an understanding of the CTI mitigation effects of background light, as well as the use of periodic charge injections to reset the illumination history in the CCDs. ${ }^{9,11}$

After launch, an analysis was made of the in-flight charge data 30 months after launch. This analysis compared the in-flight results to those obtained from the pre-flight tests and it was found that Gaia was on track to accumulate approximately 7-8 times less damage than was previously expected. ${ }^{4,12}$ In fact, the overall spacecraft performance has been so good that Gaia's nominal mission lifetime has been extended past its original 5 -year plan. While this is good news for the science goals of the mission, it is important to understand, as well as possible, the reasons behind the differences between the predicted and measured results in order to verify the reliability of pre-flight testing and to conduct more accurate testing in the future. The lower than expected CTI levels were concluded to be most likely caused by the mitigation of CTI from background straylight, a lower solar activity level during solar cycle 24 as well as the use of the $90 \%$ confidence interval. ${ }^{12}$

\section{CHARGE CALIBRATION DATA}

In order to keep track of the level of CTI and the CCD performance, calibration activities are run periodically on-board Gaia. Due to the TDI operation of the CCDs, unique strategies are implemented in order to make measurements of different detector characteristics. Analysis of these measurements have revealed that the detectors are functioning as expected with no major issues. ${ }^{4,13}$ There is still potential work to be done to accurately quantify all the factors causing the differences between the in-flight and on-ground results and to obtain a better understanding of the radiation environment.

This paper analyses the CTI results obtained by analysing the in-flight calibration data from two different points in the mission, April 2014 and December 2019. The April 2014 CTI results should be representative of 
the initial CTI state of the CCDs before launch as it is known the level of radiation in the months after launch was not at a significant enough level to impact the CTI distribution across the focal plane. The December 2019 is taken after over 5 years of orbit at L2 and with a corresponding amount of radiation damage. This is beyond the original nominal Gaia mission lifetime so it will be interesting to see how the in-flight CTI results compare to the on-ground data, given that the original end-of-mission time has been reached.

Previous measurements of the in-flight data looked at the trailing behind charge injections, a feature which is not implemented on the RVS devices as the CIs would interfere too much with the finer measurements of the RVS instruments. ${ }^{4}$ This paper analyses parallel and serial CTI measurements that were taken using unique charge calibration data, used to monitor the serial CTI. The CIs used to monitor the serial CTI have a lower injection period as compared to standard charge injections. ${ }^{10}$ The use of this data for parallel CTI measurements means that while the CTI measurements will be underestimated, due to the lower injection period, new insights are revealed about the parallel CTI in the RVS devices, which has not been analysed in much detail before. The serial CTI calibration activity was initially proposed to be run monthly or bi-monthly but due to the lower than expected amount of damage, it is now run every three to four months; it is likely to be less frequent in the future. ${ }^{4,10}$

The calibration data consists of signals at five different charge injection levels. To reliably compare the CTI measurements of the different CCDs between each other, a number of processing steps were conducted. A powerlaw relationship was fit between the parallel and serial CTI values and their corresponding charge injection levels. The reason a power law was chosen was due to a similar model used in Gaia's Charge Distortion Model (CDM). ${ }^{14}$ CDM uses an electron confinement volume model where the signal level and the volume confined by the charge cloud are related by a power-law, defined by a $\beta$ parameter. ${ }^{14}$ This relationship between CTI measurements and signal has been verified in other experimental studies ${ }^{8}$ as well with charge data from other spacecraft. ${ }^{15}$ Once this relationship was extrapolated, a CTI value was calculated for the same signal level $(10,000$ electrons was used for all the results) for all the devices. By using the same virtual signal, the CTI between all the devices can be more easily compared against each other. Apart from the Sky Mapper CCDs, data was available for all the science CCDs of the focal plane.

An important complexity should be noted about the CTI results. The parallel transfer speed is at the TDI scanning speed of $982.8 \mu$ s so CTI should be reflective of any trap species emitting around those timescales. In comparison, transfer in the serial direction takes place at two different speeds; there is a $10 \mathrm{MHz}$ flush to transfer data to the readout node and a slower $\mathrm{kHz}$ speed for readout. ${ }^{4}$ This means the charge tail will be formed from trap emission at two different timescales. For these results, only emission at $\mathrm{MHz}$ timescales is considered as it dominates the serial CTI but it is noted that it would be useful to conduct a study in the future to look at the differences between different readout speeds.

\section{CTI IN 2014}

By analysing the charge calibration data from April 2014, a few months after launch, Figure 3 was generated which highlights the serial CTI for a signal level of 10,000 electrons for all the CCDs. The calibration data from January 2014 was not used as the focal plane was known to be at a slightly higher temperature at the time. Figure 3 is similar to the plot generated in the previous set of results in 2016, verifying the reliability of these results. Assuming that charge emission from traps in the serial direction is dominated by the effects of the 10 $\mathrm{MHz}$ flush, it is suspected that the A-centre, or oxygen defect, is the primary defect responsible for these results. A previous pre-flight test revealed a trap species that emitted faster than the A-centre so there is a chance for the AC CTI to be caused from this trap species as well. ${ }^{4,16}$

The results in Figure 3 should be reflective of the initial CTI state of the CCDs as the accumulated radiation damage within the first few months is known to be minimal. The results show that the thicker red devices in both the Radial Velocity Spectrometer and the Red Photometer, all measure a lower serial CTI as compared to the other devices. While thicker devices are known to have different effects to thinner devices, such as being more sensitive to cosmic rays and having larger amounts of dark current, it is currently unknown why these devices would be manufactured measuring a lower serial CTI as compared to thinner devices with the same architecture. ${ }^{17}$ If the serial CTI is indeed primarily caused by A-centres, then this could be due to a smaller 


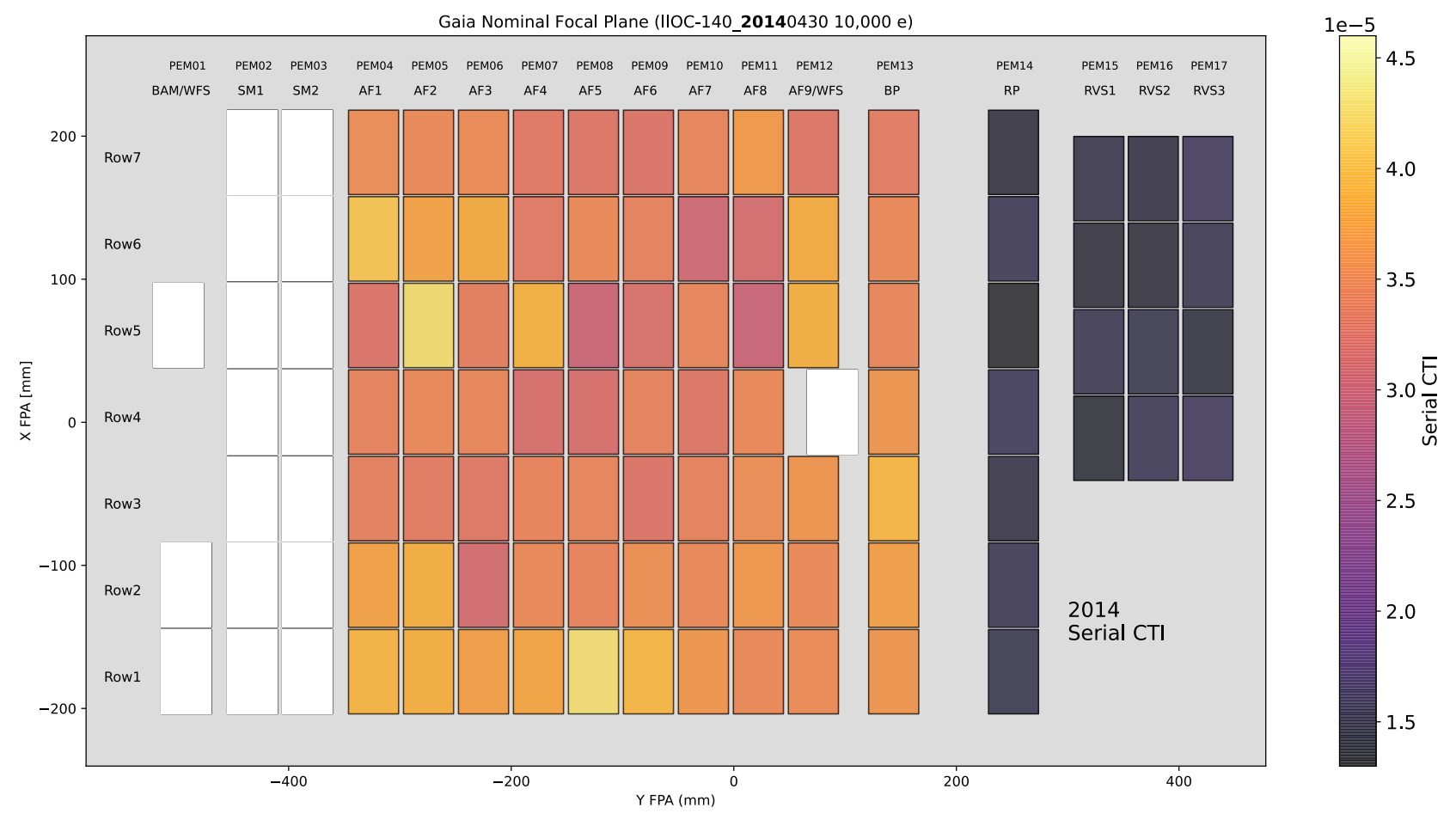

Figure 3. Distribution of the derived serial CTI values at 10000 electrons across the focal plane. This was made with data from April 2014.

amount of oxygen and A-centre defects being present in the buried channel region. This could have been the result of impurity oxygen having a larger amount of silicon to diffuse through during manufacture.

It would be useful to perform trap-pumping on unirradiated thick and thin devices to understand the trap landscape and narrow down the reasons behind this behaviour. Trap-pumping is an analysis technique that makes it possible to observe individual trap species and their properties with a high level of accuracy and quantify their densities. It involves the clocking of a flat-field signal between phases which leads to the formation of dipole maps that can be studied in more detail. ${ }^{18-20}$

As was done for the serial CTI, the parallel CTI distribution of the CCDs across the focal plane was also reproduced, as given in Figure 4. The results in Figure 4 are similar to previous CTI results that were taken for the CCDs before launch, both validating the data analysis procedure and confirming that the parallel CTI after launch is almost identical to the CTI before launch. ${ }^{10}$

It is interesting to note that some of the devices in Figure 4 measure an AL CTI that is an order of magnitude larger than as compared to the AL CTI of the other devices. Comparing the parallel CTI results to the year of manufacture in Figure 2 reveals that all the devices manufactured in 2009 measure this higher initial CTI. This means that something during the manufacturing stage has resulted in devices with a higher initial CTI; this could be due to a different silicon feedstock that was used at the time or due to some change or procedure in the manufacturing process. This does not appear to be dependent on device variant as the RP devices manufactured in earlier years have comparable AL CTI values with the other device variants. It is interesting to note that these devices do not measure these higher AC CTI results; this provides some implications about the exact defects and materials causing the higher AL CTI. It would also mean that the traps which emit in the serial and parallel directions are likely to be distinct and unique from each other.

The parallel CTI was calculated to be up to two orders of magnitude lower than the initial serial CTI. This result is likely to be reflective of the different clocking speeds in the parallel and serial directions and the 


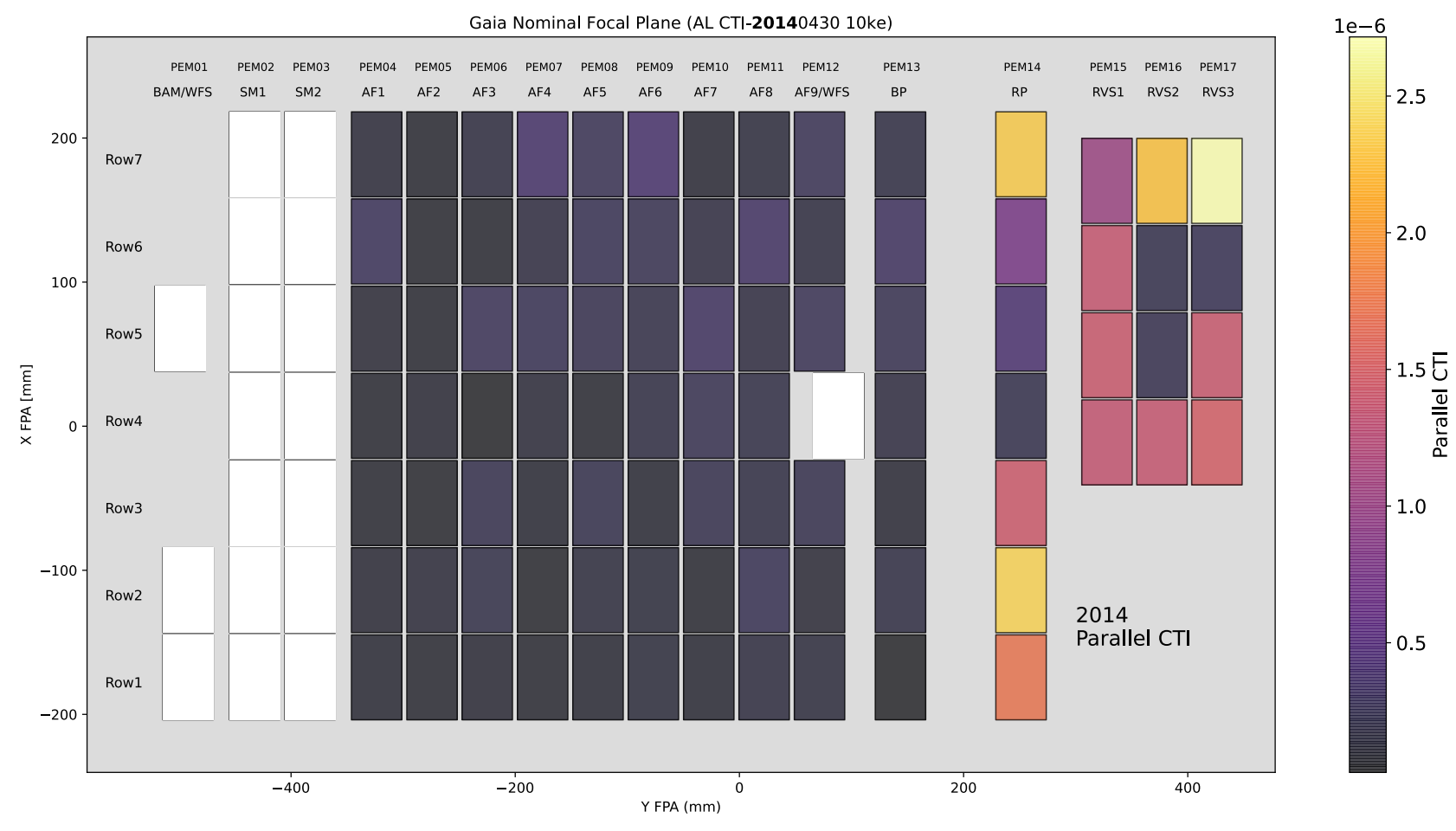

Figure 4. The distribution of the parallel CTI values, at a signal of 10000 electrons, across the focal plane as measured from the data in April 2014. A comparison of this Figure with Figure 2 reveals that the devices manufactured in 2009 have a higher initial CTI

subsequent emissions of alternate trap species as well as due to different concentrations of these alternate trap species. It appears that more manufacturing defects were present that captured and emitted on microsecond timescales as opposed to millisecond durations. The initial CTI values will be known by the industrial partners of the mission; nevertheless, care must be taken to separate the pre-launch behaviour from radiation damage. It is also recommended to use test devices and flight-model devices from the same batch, as much as feasibly possible to reduce the differences between the in-flight and pre-flight results.

\section{CTI IN 2019}

The charge calibration data from December 2019 was used to calculate the serial and parallel CTI values for each CCD. This date is several months past the original mission end-date which means that these results could be compared against previous pre-flight on-ground data to quantify the disparities between the predictions and the in-flight results. Figure 5 displays the serial CTI values across the focal plane derived from the charge data taken at the end of 2019, for a signal of 10,000 electrons. It can be seen that the pattern of serial CTI distribution across the focal plane is the same as it was in 2014. In addition to this, the serial CTI is measured to be the same order of magnitude in the 2019 data as with the 2014 data. This indicates that the serial CTI is still dominated by the initial CTI state and the amount of radiation damage has not been significant enough to alter this distribution pattern. With Gaia having been in orbit for its original nominal mission lifetime, no major deviations are seen from the previous set of CTI results and predictions, as measured in $2016 .{ }^{4}$

Figure 6 illustrates the distribution of the parallel CTI values across the focal plane, for a signal of 10,000 electrons, from the data taken in December 2019. As was the case for the serial CTI, the distribution pattern in 2019 is more reflective of the initial CTI state from 2014, with the 2009 devices still measuring larger AL CTI values than the other devices. The AL CTI values are also still measured to be an order of magnitude lower than the AC CTI values. This again suggests that the radiation damage has not been significant enough 


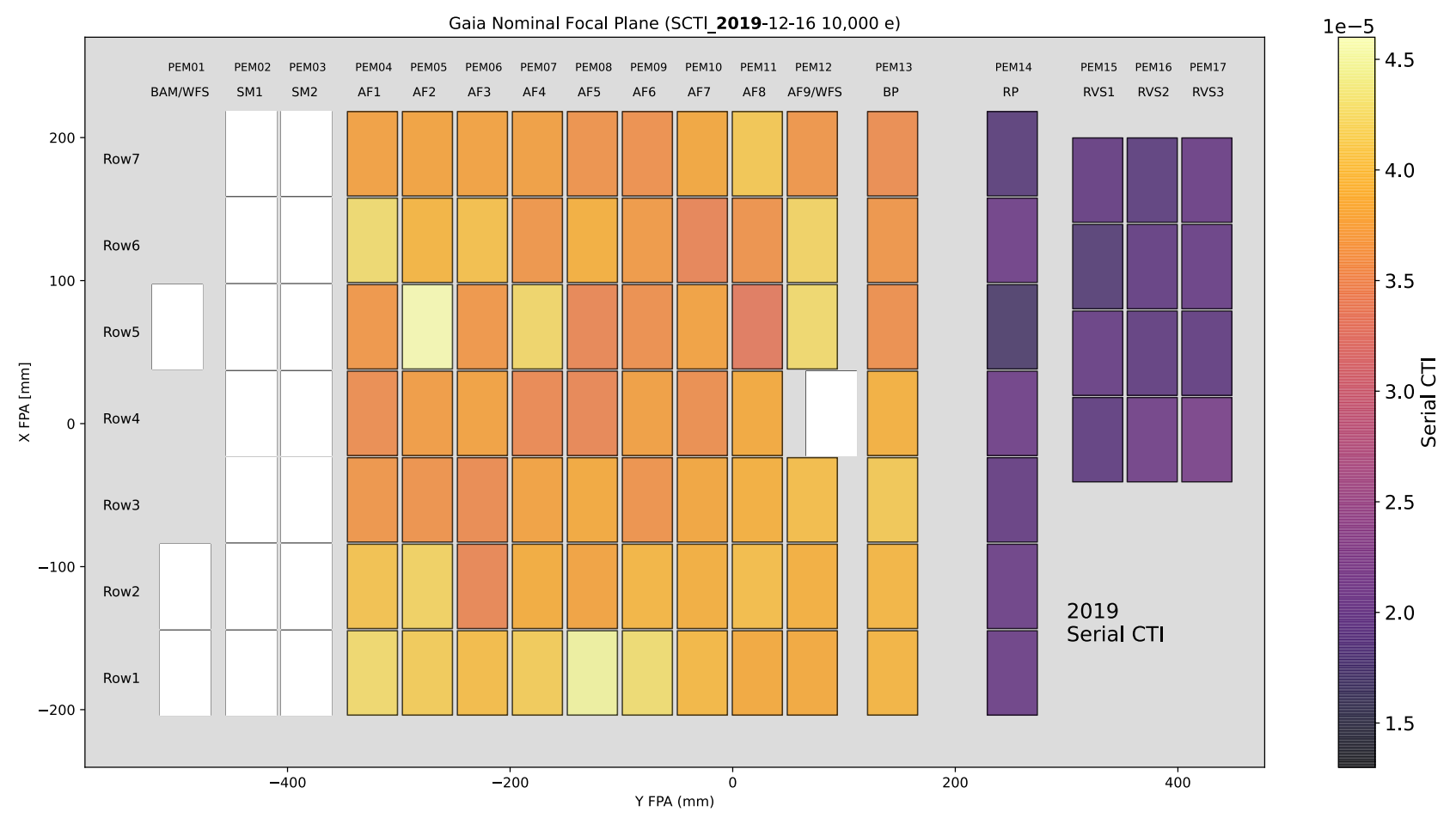

Figure 5. The distribution of the serial CTI (at 10000 electrons) across the focal plane as measured in December 2019.

to drastically alter the CTI distribution across the focal plane. In previous studies, a pattern similar to the differential shielding pattern from the pre-flight NIEL dose predictions was reflected in the magnitude CTI step increases from solar events. ${ }^{4}$ This shielding pattern is not reflected in Figure 6 which again notes the lower level of radiation as well as possibly indicates that long-term radiation radiation damage has been a result of particles with different energies than those causing the step increase. The fact that the 2009 devices still measure higher CTI values demonstrates the need to keep track of the CTI at different stages of both space missions and on-ground testing and analysis to make sure every factor is accounted for.

\section{INCREASE IN CTI}

The mean serial CTI values for each different instrument, for a given signal level (10,000 electrons), can be calculated from the April 2014 and December 2019 datasets; Table 1 details these mean serial CTI values. It also contains the calculated values for the difference in serial CTI (representing the increase), the percentage increase as well as the increase with respect to time. It is noted that the percentage increases are much larger for the thicker red devices than for the other device variants. Most of this larger percentage increase value however, can be attributed to the lower CTI values from 2014.

From Table 1, it is noted that the increase in the physical CTI values between 2014 and 2019 is higher for the red devices than the other devices variants. Some physical factor is causing the thicker red devices to increase in CTI faster as compared to the other ones. The data is not precise enough to pinpoint the exact reason behind this although several different hypotheses are proposed. These include the lower initial serial CTI, the geometry of the thicker devices themselves affecting the rate of radiation damage or the interaction of the incoming particles with the shielding and optics. Despite this increased rate of damage, it is still the same order of magnitude as it is for the other devices. The AC CTI in the red devices is still measured to be lower than that of the other device variants after more than 5 years of orbit. This is attributed to the very low rate of CTI increase, as noted by the increase in CTI values with respect to time from Table 1. 


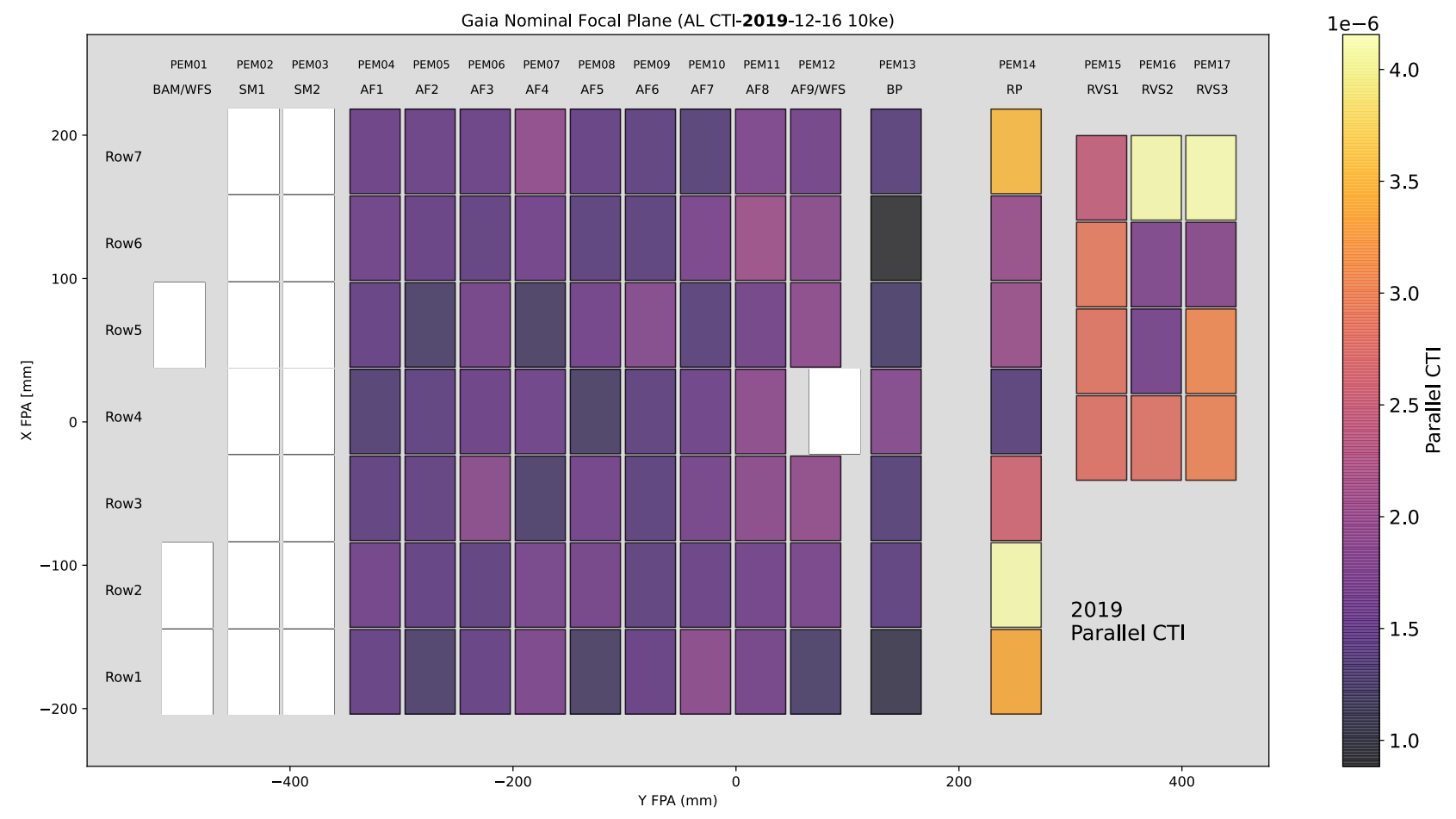

Figure 6. The distribution of the parallel CTI (at 10000 electrons) across the focal plane as measured in December 2019.

Table 2 details the mean parallel CTI values as measured in 2014 and 2019 as well as the increase in CTI, both physically and with respect to time (for a signal of 10,000 electrons). Due to the 2009 red devices measuring a larger initial CTI, the RP devices have been split by their year of manufacture in this analysis. Like the serial CTI results, it is noted that the parallel CTI levels in 2019 for the 2009 devices are the same order of magnitude as they were in 2014. The parallel CTI in the other devices increases by an order of magnitude however, this is attributed to the comparatively lower initial CTI levels in 2014. Its is noted that the increase of CTI has been approximately the same for all the devices, with respect to a given uncertainty.

Table 1. The mean serial CTI for each instrument as measured in April 2014 and December 2019 as well as the absolute increase and percentage increase in CTI and increase in CTI with time. All these values are for a signal level of 10,000 electrons.

\begin{tabular}{|l|l|l|l|l|l|}
\hline $\begin{array}{c}\text { CCD Instru- } \\
\text { ments }\end{array}$ & $\begin{array}{l}2014 \text { Mean AC } \\
\text { CTI }\left(\times 10^{-5}\right)\end{array}$ & $\begin{array}{l}2019 \text { Mean AC } \\
\text { CTI }\left(\times 10^{-5}\right)\end{array}$ & $\begin{array}{l}\text { CTI Increase } \\
\left(\times 10^{-5}\right)\end{array}$ & $\begin{array}{l}\text { CTI Increase } \\
(\%)\end{array}$ & $\begin{array}{l}\text { CTI Increase/Year } \\
\left(\times 10^{-7}\right)\end{array}$ \\
\hline $\mathrm{AF}$ & $3.5 \pm 0.3$ & $3.8 \pm 0.3$ & $0.3 \pm 0.6$ & 9 & $6 \pm 10$ \\
\hline $\mathrm{BP}$ & $3.6 \pm 0.2$ & $3.8 \pm 0.2$ & $0.2 \pm 0.3$ & 8 & $4 \pm 5$ \\
\hline $\mathrm{RP}$ & $1.5 \pm 0.1$ & $1.9 \pm 0.1$ & $0.4 \pm 0.1$ & 27 & $7 \pm 2$ \\
\hline RVS & $1.5 \pm 0.1$ & $1.9 \pm 0.1$ & $0.4 \pm 0.1$ & 27 & $7 \pm 2$ \\
\hline
\end{tabular}

It is noted that both the yearly increase in CTI for the parallel and serial directions is the same order of magnitude. This gives more credence to the notion that the traps responsible for parallel and serial CTI are distinct and are forming at approximately equal rates. This is an unsurprising result given that the transfer speeds in both the parallel and serial directions are unique and were chosen in conjunction with the operating temperature of the spacecraft to minimise the effects of CTI in both directions. The order of magnitude of the 
yearly increase in CTI is lower than both the initial parallel and serial CTI levels. This verifies the observations of a low-activity solar cycle which means fewer particles have been causing radiation damage. It has been noted previously that there is an anti-correlation between the solar activity and the impact of Galactic Cosmic Rays (GCRs) but from these results, it would appear that the impact of GCRs has not been very substantial either. ${ }^{12}$ The differing initial CTI levels do not appear to have any impact on the amount of CTI increase although this is an expected result as initial CTI is caused by traps formed from manufacturing which are distinct to the traps formed from radiation. The initial serial CTI is the only potential point of contention though other physical reasons could explain these results as well.

Table 2. The mean parallel CTI for each instrument as measured in April 2014 and December 2019 as well as the absolute increase and percentage increase in CTI and increase in CTI with time. All these values are for a signal level of 10,000 electrons.

\begin{tabular}{|l|l|l|l|l|}
\hline CCD Instruments & $\begin{array}{l}\text { 2014 Mean AL } \\
\text { CTI }\left(\times 10^{-6}\right)\end{array}$ & $\begin{array}{l}\text { 2019 Mean AL } \\
\text { CTI }\left(\times 10^{-6}\right)\end{array}$ & $\begin{array}{l}\text { CTI Increase } \\
\left(\times 10^{-6}\right)\end{array}$ & $\begin{array}{l}\text { CTI Increase/Year } \\
\left(\times 10^{-7}\right)\end{array}$ \\
\hline $\mathrm{AF}$ & $0.2 \pm 0.1$ & $1.6 \pm 0.2$ & $1.4 \pm 0.2$ & $2.5 \pm 0.4$ \\
\hline $\mathrm{BP}$ & $0.2 \pm 0.1$ & $1.3 \pm 0.3$ & $1.1 \pm 0.3$ & $2.0 \pm 0.6$ \\
\hline $\mathrm{RP}($ Pre-2009) & $0.4 \pm 0.2$ & $1.8 \pm 0.2$ & $1.4 \pm 0.3$ & $2.5 \pm 0.7$ \\
\hline $\mathrm{RP}(2009)$ & $1.7 \pm 0.5$ & $3.2 \pm 0.6$ & $1.5 \pm 0.8$ & $2.7 \pm 1.5$ \\
\hline
\end{tabular}

\section{FUTURE WORK AND CONCLUSIONS}

This paper has presented an overview of the CTI measurements on the Gaia CCDs a few months after launch and after over 5 years of orbit. Interesting results and observations have been revealed between the CTI of the Gaia CCDs and the radiation damage that they have experienced. Different devices at the beginning of the mission were measured to have different amounts of initial CTI. All the red devices measured a lower serial CTI by a factor of approximately 2 while the red devices manufactured in 2009 measured a higher parallel CTI. The current physical reasoning behind these results is currently unknown but it serves as a useful starting point for future investigations. It is recommended to perform an analysis of devices that reveals details about the defect concentrations like trap-pumping. Performing trap-pumping on different device variants will provide more quantitative details on how these variants are distinct from each other.

The increase of CTI is measured to be the same order of magnitude in the parallel and serial directions, around $10^{-7}$ a year. As outlined previously, this would suggest that the trap species responsible for parallel and serial CTI are unique and not competing against each other. Interestingly, the thicker red devices seem to measure a larger increase in serial CTI as compared to the other device variants whereas the increase in parallel CTI is more equivalent to that of the variants. It is currently unknown why this might be, with the current hypotheses suggesting that it could be related to the geometry of the devices, the initial CTI affecting the increase of CTI or due to interactions between the incoming radiation and the optics set up in front of the devices. This lower order of magnitude CTI increase in both directions, as compared to the order of magnitudes of the initial CTI, once again verifies that CCDs have been accumulating less damage than was predicted and there has been no significant deviations from the extrapolations of the measurements of the previous in-flight data by Crowley et. al (2016). ${ }^{12}$ These low levels of damage means that Gaia should have no trouble operating with its mission extensions into 2025.

Despite the innovative results that have been revealed from this investigation, there is still further room for more analysis. The results described in the paper only used the charge calibration datasets taken from two points in the mission, April 2014 and December 2019. It is possible that outlier effects may be present in these datasets which could skew the results in different ways. Furthermore, only using two datasets means that the uncertainties have been relatively high. This is particularly obvious in the case for the increase in serial CTI where it can be seen in Table 1 that the uncertainties for the increase in CTI for the AF and BP devices is larger than the increase itself. 
A future paper will present the analysis of all the charge calibration data, from the beginning of the mission to December 2019. Using all the data, a more accurate value for the rate of increase of CTI across all the devices and instruments can be extrapolated. It is also known that the $10 \mathrm{MeV}$ equivalent dose is predicted to be different across the focal plane for different devices. ${ }^{4,10}$ It would be interesting to see the results after accounting for this alternate NIEL dose to see how differently or similarly different devices across the focal plane experience radiation damage. A more in-depth analysis and comparison will also be performed between the CTI results from the in-flight data and the on-ground results. By studying this as well as looking into the differences between the predicted solar activity before launch and the actual activity during orbit, more insights will be revealed into the nature of the radiation damage of the Gaia CCDs and the reasons behind the disparities between the pre-flight tests and in-flight measurements. The results from these investigations can provide further insights into the radiation environment of L2 and subsequent radiation damage levels and positively feedback into the studies and sector analysis for other missions.

\section{ACKNOWLEDGMENTS}

This work was carried out at the Center for Electronic Imaging; it would not have been made possible without access to non-scientific in-flight data made available from the European Space Agency through a Networking Partnering Initiative. Additional thanks is given to the Science and Technology Facilities Council for their funding of the studentship with further thanks to Teledyne $\mathrm{e} 2 \mathrm{v}$ for sponsoring the CASE studentship.

\section{REFERENCES}

[1] Perryman, M., "Overview of the Gaia mission," in [The Three-Dimensional Universe with Gaia], 576, 15 (2005).

[2] Prusti, T., De Bruijne, J., Brown, A. G., Vallenari, A., Babusiaux, C., Bailer-Jones, C., Bastian, U., Biermann, M., Evans, D., Eyer, L., et al., "The Gaia mission," Astronomy 8 Astrophysics 595, A1 (2016).

[3] Gaia, C., Brown, A., Vallenari, A., Prusti, T., de Bruijne, J., Babusiaux, C., Juhász, Á., Marschalkó, G., Marton, G., Molnár, L., et al., "Gaia Data Release 2 Summary of the contents and survey properties," Astronomy $\mathcal{E}$ Astrophysics 616(1) (2018).

[4] Crowley, C., Kohley, R., Hambly, N. C., Davidson, M., Abreu, A., Van Leeuwen, F., Fabricius, C., Seabroke, G., de Bruijne, J., Short, A., et al., "Gaia data release 1-On-orbit performance of the Gaia CCDs at L2," Astronomy 83 Astrophysics 595, A6 (2016).

[5] Seabroke, G., Holland, A., and Cropper, M., "Modelling radiation damage to ESA's Gaia satellite CCDs," in [High Energy, Optical, and Infrared Detectors for Astronomy III], 7021, 70211P, International Society for Optics and Photonics (2008).

[6] Seabroke, G., Prod'homme, T., Murray, N., Crowley, C., Hopkinson, G., Brown, A., Kohley, R., and Holland, A., "Digging supplementary buried channels: investigating the notch architecture within the CCD pixels on ESA's Gaia satellite," Monthly Notices of the Royal Astronomical Society 430(4), 3155-3170 (2013).

[7] Janesick, J. R., [Scientific charge-coupled devices], vol. 83, SPIE press (2001).

[8] Hall, D. J., Bush, N., Murray, N., Gow, J., Clarke, A., Burgon, R., and Holland, A., "Challenges in photonstarved space astronomy in a harsh radiation environment using CCDs," in [UV/Optical/IR Space Telescopes and Instruments: Innovative Technologies and Concepts VII], 9602, 96020U, International Society for Optics and Photonics (2015).

[9] Prod'homme, T., From electrons to stars : modelling and mitigation of radiation damage effects on astronomical CCDs, PhD thesis, Leiden University (2011).

[10] Crowley, C., "An assessment of CCD radiation damage at launch +25 months," Tech. Rep. GAIA-CH-TNESA-CMC-017-1, ESAC (03 2016).

[11] Brown, S. W., Characterization and mitigation of radiation damage on the Gaia astrometric field, $\mathrm{PhD}$ thesis, University of Cambridge (2009).

[12] Crowley, C., Abreu, A., Kohley, R., Prod'homme, T., and Beaufort, T., "Radiation effects on the Gaia CCDs after 30 months at L2," in [High Energy, Optical, and Infrared Detectors for Astronomy VII], 9915, 99150K, International Society for Optics and Photonics (2016). 
[13] Cropper, M., Katz, D., Sartoretti, P., Prusti, T., De Bruijne, J., Chassat, F., Charvet, P., Boyadjian, J., Perryman, M., Sarri, G., et al., "Gaia Data Release 2-Gaia Radial Velocity Spectrometer," Astronomy \& Astrophysics 616, A5 (2018).

[14] Short, A., Crowley, C., de Bruijne, J. H., and Prod'homme, T., "An analytical model of radiation-induced Charge Transfer Inefficiency for CCD detectors," Monthly Notices of the Royal Astronomical Society 430(4), 3078-3085 (2013).

[15] Ryon, J. and Grogin, N., "ACS/WFC Parallel CTE from EPER Tests," Tech. Rep. ACS 2018-09, The Association of Universities for Research in Astronomy (12 2018).

[16] Hopkinson, G. R., Short, A., Vetel, C., Zayer, I., and Holland, A. D., "Radiation effects on astrometric CCDs at low operating temperatures," IEEE transactions on nuclear science 52(6), 2664-2671 (2005).

[17] Holland, S. E., Groom, D. E., Palaio, N. P., Stover, R. J., and Wei, M., "Fully depleted, back-illuminated charge-coupled devices fabricated on high-resistivity silicon," IEEE Transactions on Electron Devices 50(1), $225-238$ (2003).

[18] Hall, D. J., Murray, N. J., Holland, A. D., Gow, J., Clarke, A., and Burt, D., "Determination of in situ trap properties in CCDs using a "single-trap pumping" technique," IEEE Transactions on Nuclear Science 61(4), 1826-1833 (2014).

[19] Wood, D., Hall, D., Murray, N., Gow, J., Holland, A., Turner, P., and Burt, D., "Studying charge-trapping defects within the silicon lattice of a p-channel CCD using a single-trap "pumping"technique," Journal of Instrumentation 9(12), C12028 (2014).

[20] Murray, N. J., Burt, D. J., Hall, D., and Holland, A. D., "The relationship between pumped traps and signal loss in buried channel CCDs," in [UV/Optical/IR Space Telescopes and Instruments: Innovative Technologies and Concepts VI], 8860, 88600H, International Society for Optics and Photonics (2013). 